\title{
Demonstration of an All-Fiber Ultra-Low Numerical Aperture Ytterbium-Doped Large Mode Area Fiber in a Master Oscillator Power Amplifier Configuration Above $1 \mathrm{~kW}$ Power Level
}

\author{
Yakup Midilli ${ }^{\circledR}$, Member, OSA and Bülend Ortaç ${ }^{\circledR}$, Senior Member, OSA
}

\begin{abstract}
We demonstrate an all-fiber ultra-low numerical aperture high power fiber laser system operating in the continuouswave regime at a central wavelength of $1080 \mathrm{~nm}$. A special Ytterbium-doped fiber preform has been designed and fabricated by using modified chemical vapor deposition technique with the deposition percentages of $0.03 \mathrm{~mol} \%$ of $\mathrm{Yb}_{2} \mathrm{O}_{3}, 1.8 \mathrm{~mol} \%$ of $\mathrm{Al}_{2} \mathrm{O}_{3}$, and $2.1 \mathrm{~mol} \%$ of $\mathrm{P}_{2} \mathrm{O}_{5}$. Then it has been drawn to obtain an active fiber having core/cladding diameters of $26 \mu \mathrm{m} / 410 \mu \mathrm{m}$ respectively. The numerical aperture of the fiber has been first predicted as 0.034 from refractive index profile of the preform. Afterward, this number has been verified with a simple test setup by altering the bending diameter of the fiber and the excitation conditions of it. To test the high power performance, a laser system has been constructed in a master oscillator power amplifier configuration, and $\sim 80 \mathrm{~W}$ seed signal power is amplified to $1.05 \mathrm{~kW}$. The quality of the laser output beam has been measured in terms of $\mathrm{M}^{2}$ value along both $\mathrm{x}$ and $\mathrm{y}$ coordinates as $\mathbf{1 . 1 1}$ and 1.16 respectively.
\end{abstract}

Index Terms-Low numerical aperture, MOPA configuration, optical fiber fabrication, optical fibers, optical fiber lasers, specialty fibers.

\section{INTRODUCTION}

$\mathbf{F}$ IBER lasers have been widely used in the field of industry as well as the health and defense applications due to their critical features such as robustness, compactness and directionality. It has been possible to achieve $\mathrm{kW}$ class fiber laser systems after the developments in the optical fiber technology introducing the generation of Large Mode Area (LMA) fibers with low NA values $\sim 0.06[1]-[4]$. This breakthrough has provided with the use of fiber lasers in the defense industry. The fabrication of these silica glass-based LMA optical fibers can be performed with the use of the Modified Chemical Vapor Deposition (MCVD) technique [5], [6]. High purity and high-quality preforms can easily be manufactured by using this technique since the chemicals

Manuscript received October 2, 2019; revised December 22, 2019 and January 21, 2020; accepted January 24, 2020. Date of publication January 29, 2020; date of current version April 1, 2020. This work was supported in part by The Scientific and Technological Research Council of Turkey (TUBÍTAK) (Corresponding author: Bülend Ortaç.)

The authors are with the UNAM - National Nanotechnology Research Center and Institute of Materials Science and Nanotechnology, Bilkent University, Bilkent, Ankara 06800, Turkey (e-mail: yakup.midilli@bilkent.edu.tr; ortac@bilkent.unam.edu.tr).

Color versions of one or more of the figures in this article are available online at https://ieeexplore.ieee.org.

Digital Object Identifier 10.1109/JLT.2020.2970213 used have the purity level of $5 \mathrm{~N}$ or $6 \mathrm{~N}$. Therefore, the LMA fibers used in defense application in the multi-kW power levels have been preferred to be manufactured with this method since the impurity level gains importance when the power scales up. Moreover, it is possible to monitor and control both the amount and flow of the chemicals, which is an important fact to lower the difference between the refractive indices of the core and inner cladding regions for the fabrication of low NA optical fibers [7]. This process is very challenging even by the MCVD system due to the fact that several precursors with different deposition efficiencies get in a competition within the silica host matrix, so that requiring a great effort to successfully place different dopants in the host matrix for the adjustment of the desired refractive index profile (RIP). LMA fibers have larger core diameters than those of intrinsically single-mode fibers with corresponding higher optical power damage and nonlinear effect thresholds. Depending on how large the core diameter is, LMA fibers supporting more than one mode are also called as few-mode optical fibers, which imply that, in addition to the fundamental mode, higher-order modes (HOMs) also survive inside the optical fiber. Thus, the presence of HOMs inside the optical fiber decreases the quality of the laser output beam dramatically. Besides, the presence of HOMs can cause some other problems over certain pump power threshold level such as Transverse Modal Instability (TMI) which has a thermal origin and said to be caused by the Stimulated Thermal Rayleigh Scattering (STRS) or by thermal mode coupling processes alternatively [8]-[11]. In the presence of TMI, the total energy in the fundamental mode couples to the HOMs and then couples back to the fundamental mode in a repeating manner. Therefore, not to confront such an undesirable situation, HOMs should be suppressed inside the fiber so that only the fundamental mode can survive. The most fundamental and important method to get rid of the HOMs is the bend-induced loss mechanism in which the fiber is bent at a certain diameter so that HOMs cannot propagate inside the fiber anymore. Another approach is to design and fabricate a new type of large core active fiber to stop the intrinsic formation and propagation of HOMs inside the fiber. In this new approach, even though the NA value of the fiber is lowered, the fiber only supports the propagation of the fundamental mode with a larger core size. 
In the literature, an active fiber having ultra-low $(<0.04) \mathrm{NA}$, was proposed [12], [13] in 2009. Additionally, the authors of Ref. [8] showed a low NA fiber laser having an output power over $2.1 \mathrm{~kW}$ with a high beam quality of $\mathrm{M}^{2}=1.2$. The same group also demonstrated a laser having a beam quality of $\mathrm{M}^{2}$ $=1.1$ by designing a low NA fiber $(\mathrm{NA}=0.038)$ with $35 \mu \mathrm{m}$ core diameter [9]. In Ref. [14], systematic numeric studies were performed to simulate laser output characteristics by varying the NA (0.025-0.05), core diameter $(20 \mu \mathrm{m}-100 \mu \mathrm{m})$ and the bending diameter $(0.4 \mathrm{~m}-1.6 \mathrm{~m})$ of the fiber. Afterward, a $3 \mathrm{~kW}$ output power level was achieved by designing a new low NA fiber [15], [16]. The highest power level in the literature, which is $4.3 \mathrm{~kW}$, had finally been achieved by Tünnermann's group [17]. However, all of these works based on free space orientation; that is to say, pump light is coupled to the active fiber via free space with the help of several lens and objective combinations. Recently, all-fiber ultra-low NA fiber laser studies started to be published in a resonator configuration [18] and as a seed source in an amplifier system [19]. However, in this work, we demonstrate a monolithic all-fiber high power fiber Master Oscillator Power Amplifier (MOPA) system based on an ultra-low NA ytterbium (Yb)-doped active fiber having $26 \mu \mathrm{m} / 410 \mu \mathrm{m}$ core/cladding diameters respectively with an NA of 0.034 which has been verified experimentally. Yb-doped preforms were fabricated by a conventional Modified Chemical Vapor Deposition (MCVD) technique. All starting raw chemicals used in this system were purchased with high purity levels of $5 \mathrm{~N}$ (99.999\%) and 6N (99.9999\%). A Heraeus branded F300 quartz substrate tube whose inner diameter is $24 \mathrm{~mm}$ and the outer diameter is $28 \mathrm{~mm}$, was used for the deposition at $1930{ }^{\circ} \mathrm{C}$ via an $\mathrm{H}_{2} / \mathrm{O}_{2}$ torch. Then, the substrate tube was collapsed at $2050^{\circ} \mathrm{C}$ by a furnace. Upon obtaining the preform, the core/clad ratio was adjusted by the post-processes such as stretching and jacketing using the MCVD system as well. After manufacturing process, characterizations of the final preform were conducted using a Polariscope to observe the discrepancies if exists any in the core region, an optical microscope Zeiss Axio Vert A1 to see the cross-section, a P102 Photon Kinetics preform analyzer to measure the refractive index profile (RIP). Then it was drawn by an OptoGear branded fiber drawing tower and the drawn fiber was coated with primary and secondary polymers to make it bendable.

\section{Preform AND Fiber Properties}

Fig. 1 shows the refractive index profile (RIP) of the manufactured preform which has been measured by a so-called preform analyzer system operating at $633 \mathrm{~nm}$ wavelength. For that purpose, a preform slice with a thickness less than $4 \mathrm{~mm}$ was used in this measurement. The RIP of the nearly whole preform $(17.4 \mathrm{~mm})$ was shown in Fig. 1(a) in which the core region was pointed out. To investigate the core region deeply, the zoomed-in version of Fig. 1(a) was sketched as in Fig. 1(b).

It is a well-known fact that $\mathrm{Yb}^{3+}, \mathrm{Al}^{3+}$ and $\mathrm{P}^{5+}$ ions lead to increase the refractive index whereas $\mathrm{Al}^{3+}$ and $\mathrm{P}^{5+}$ ions can yield to formation of $\mathrm{AlPO}_{4}$ structural units within the silica host matrix.
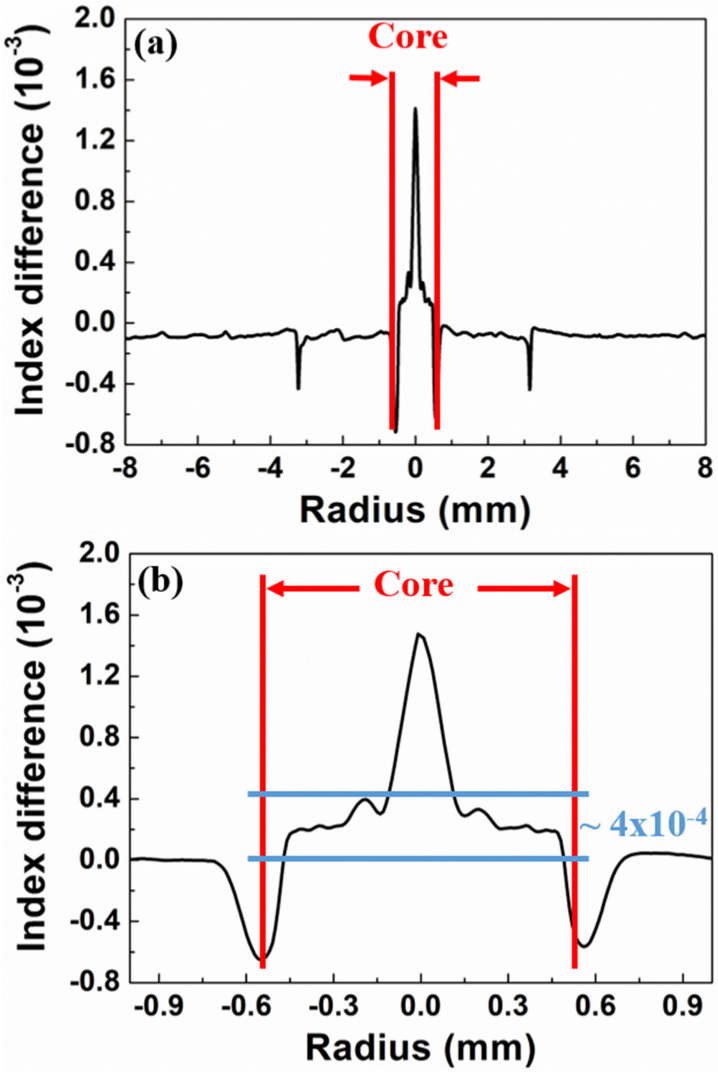

Fig. 1. (a) Refractive index profile (RIP) of the nearly whole preform and (b) the core region of it.

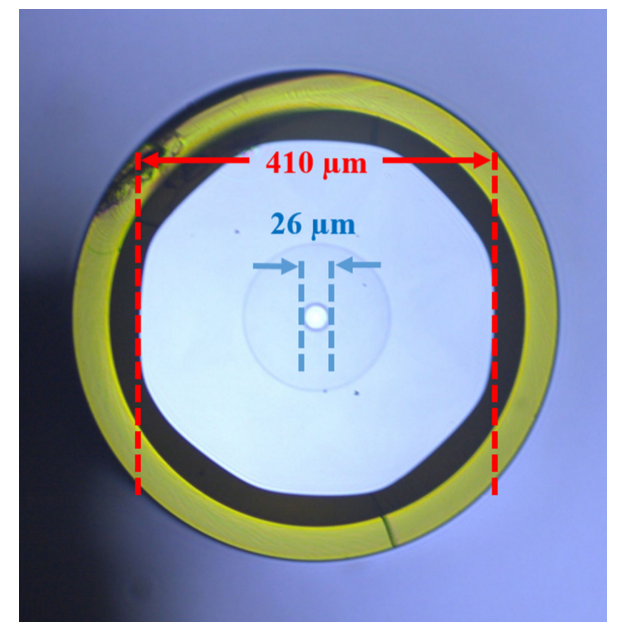

Fig. 2. Optical microscope image of the cross section of the drawn fiber.

Moreover, it has been aimed to dope the silica glass with equimolar amounts of $\mathrm{Al}_{2} \mathrm{O}_{3}$ and $\mathrm{P}_{2} \mathrm{O}_{5}$ so that the $\mathrm{AlPO}_{4}$ formation help to reduce the refractive index [20], [21].

Therefore, this approach could be utilized to fabricate the ultra-low NA fibers. Benefitting from this fact, we have also tried to dope $\mathrm{Al}^{3+}$ and $\mathrm{P}^{5+}$ ions as equal as possible in addition to a decrease in the $\mathrm{Yb}^{3+}$ ion concentration in the core region in a controlled manner. After the fabrication, we have 

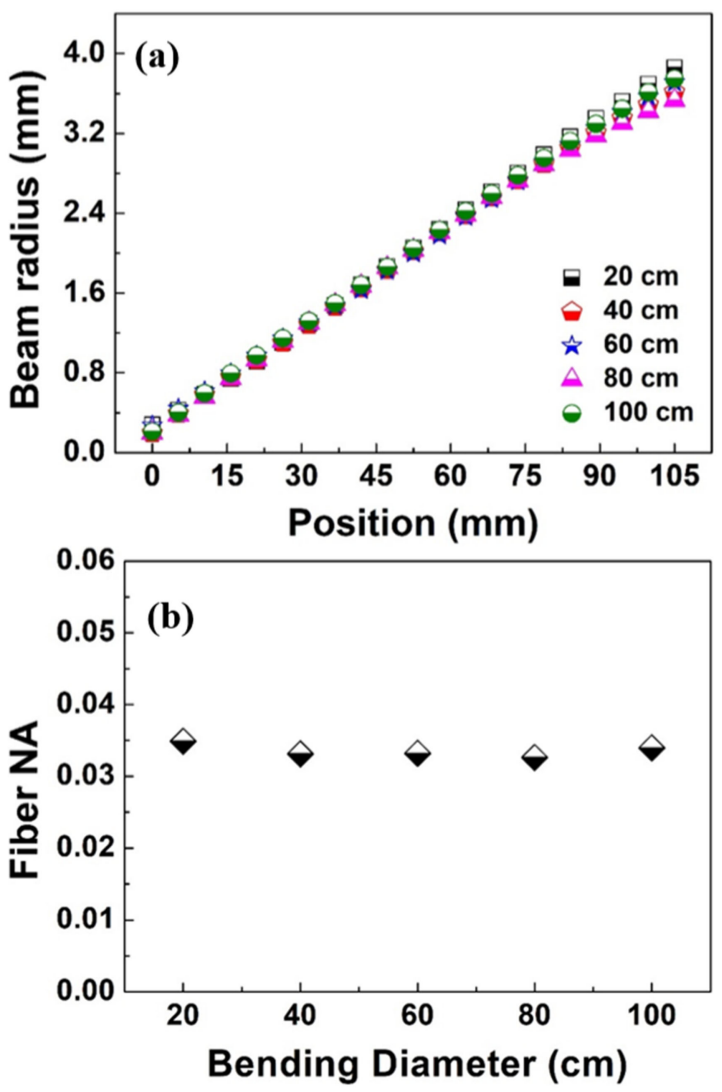

Fig. 3. (a) Beam divergence measurement of the fiber with respect to the bending diameter and (b) the corresponding NA values.

performed Wavelength-Dispersive X-Ray Spectroscopy (WDS) to determine the elemental ratio of the fiber preform.

Based on the WDS measurement, the molar ratio of the $\mathrm{Yb}_{2} \mathrm{O}_{3}, \mathrm{Al}_{2} \mathrm{O}_{3}$ and $\mathrm{P}_{2} \mathrm{O}_{5}$ have been found as $0.03 \mathrm{~mol} \%$, $1.8 \mathrm{~mol} \%$ and $2.1 \mathrm{~mol} \%$ respectively. However; in the contrary to our intention there existed an undesired peak at the center of the core region of the preform, which has been created during the collapse pass of the manufacturing process of the preform. Since the homogeneity of the RIP of the preform has been deteriorated by the peak at the center, taking into consideration of this fact, we have predicted and claimed that the average index difference of the whole core region might be calculated as $4 \times$ $10^{-4}$ as indicated in Fig. 1(b). By a simple calculation, such index difference would correspond to an NA value of 0.034 theoretically. After the prediction of the NA of the preform, full attention has been focused on the physical dimensions of the drawn fiber. Therefore, a piece of the fiber was cleaved at an angle close to zero with its polymer coating onside, and an optical microscope image of the cross-section of the drawn fiber has been taken and illustrated as shown in Fig. 2. The core and the cladding diameters of the ultra-low NA active fiber have been measured as $26 \mu \mathrm{m}$ and $410 \mu \mathrm{m}$ respectively. Additionally, the diameter of the fiber reaches up to $550 \mu \mathrm{m}$ with its polymer coating. Since we have a predicted NA value on hand, we can also calculate the $\mathrm{V}$ number of the fiber easily just for the sake of getting an idea about the modal content of this fiber. $V$ number depends on the NA and the core radius of the fiber. If the $\mathrm{V}$
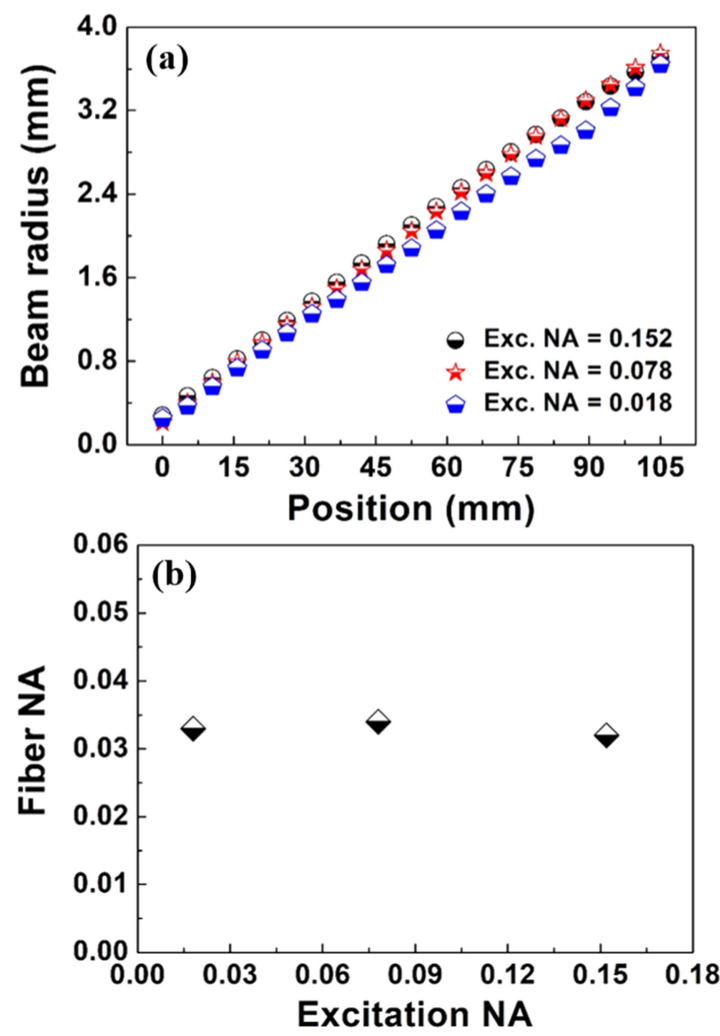

Fig. 4. (a) Beam divergence measurement of the fiber with respect to the excitation NA and (b) the corresponding NA values.

number of fiber is smaller than the threshold value of 2.405 then it is said that the fiber is intrinsically single mode which means that there can be no HOMs to survive in this fiber.

When we calculate the $\mathrm{V}$ number of this fiber according to the equation above, with a predicted NA value of 0.034 , the core radius of $13 \mu \mathrm{m}$ and finally assuming the wavelength is $1.08 \mu \mathrm{m}$, we come up with a number of 2.57 which is relatively a bit larger but still very close to the threshold value. Therefore, we can easily conclude that the fiber reported in this study is considered to be not an intrinsically single-mode one. Nevertheless, HOMs can be eliminated even with a large bending diameter of the fiber.

\section{VERIFICATION OF THE NUMERICAL APERTURE OF THE FIBER}

Henceforth, we verify the NA of the fiber experimentally which has been previously predicted from the RIP of the preform. For this purpose, we have built an experimental setup to measure the NA of the fiber directly. The setup consisted of a laser source operating at $1200 \mathrm{~nm}$ central wavelength, chosen specifically due to the less absorption of $\mathrm{Yb}$ atoms at that wavelength region, and a Thorlabs F230APC-1064 fibercoupled collimator having an NA of 0.55 and focal length of $4.56 \mathrm{~mm}$, to collimate the outgoing laser light. In free space, finally a lens was used to focus the laser light into the fiber. At the end of the setup, a Thorlabs BP109-IR beam profiler was utilized to measure the NA of the fiber. We have taken an $8 \mathrm{~m}$ long piece of the fiber varying the bending diameter and the excitation NA of the laser and measured its NA with respect to 


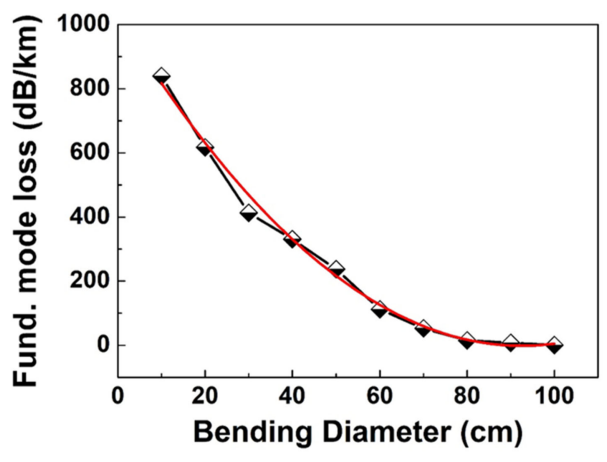

Fig. 5. Fundamental mode loss measurement of the fiber.

the source.Firstly, we have performed the NA measurement by changing the bending diameter of the fiber from $100 \mathrm{~cm}$ to $20 \mathrm{~cm}$ to observe the bending effect on the NA of the fiber while the excitation NA is fixed at 0.078 by implementing a lens having $8 \mathrm{~mm}$ focal length right after the collimator. The excitation NA was chosen larger than our prediction of the fiber NA to make sure that the whole fiber was fulfilled so that to be able to measure directly the NA of the fiber rather than the divergence of the light passing through. Under these circumstances, the divergence data that we have obtained from the beam profiler are as in Fig. 3(a). The divergence angles corresponding to each bending diameter are $3.799^{\circ}, 3.735^{\circ}, 3.803^{\circ}, 3.794^{\circ}$, and $3.995^{\circ}$ respectively. By taking the sine of the half of these divergence angles for each bending diameter, we have obtained the fiber NA values as shown in Fig. 3(b). The fiber NA values obtained with respect to the bending diameters of the fiber are $0.0348,0.0331$, $0.0332,0.0326$ and 0.0339 correspondingly. The average NA value of these 5 different NA values is then found as 0.0335 .

After measuring the NA of the fiber by varying the bending diameter, we have examined the effect of the excitation NA on the fiber NA itself. For that purpose, we have changed the lens after the collimator to adjust the NA of the laser source that is going to pass through the fiber. We had 3 pieces of bi-convex lenses having $4.2 \mathrm{~mm}, 8 \mathrm{~mm}$ and $35 \mathrm{~mm}$ focal lengths to alter the excitation NA. Based on the values of each lens, the excitation NAs have been calculated as $0.152,0.078$ and 0.018 respectively. At these three different scenarios, the NA measurement has been repeated and the results are as in Fig. 4(a). The corresponding fiber NA values have been obtained as $0.033,0.034$ and 0.032 as shown in Fig. 4(b). Then, the average NA value based on these different excitation NAs is found as 0.033 . Based on this information that we have obtained from all of the measurements, it can easily be concluded that no matter how the excitation NA was changed the NA of this fiber is indeed around 0.034. In addition to the NA verification measurement, the fundamental mode loss of the fiber has also been studied. For that purpose, we have installed a simple setup consisting of a laser source operating at $1200 \mathrm{~nm}$ wavelength range and a bi-convex lens to focus the light directly into the fiber and a pinhole to prevent the light coming from the cladding region of the fiber.

The laser source has been kept constant during the whole measurement and the lens has not been touched after the optimal coupling condition has been satisfied to ensure that the only variable in this measurement would be the bending diameter of the fiber.

The bending diameter of the fiber has been altered from $100 \mathrm{~cm}$ to $10 \mathrm{~cm}$ to observe the loss of the fundamental mode of the fiber precisely. The data obtained from this measurement has been plotted as shown in Fig. 5. Based on this data, the fundamental mode loss dramatically increases beyond the $50 \mathrm{~cm}$ bending diameter. Therefore, it can easily be concluded that the critical bending diameter for this fiber is around $50 \mathrm{~cm}$.

\section{High Power Performance Test RESULTS OF THE FIBER}

We have tested the high power performance of the fiber by installing a MOPA configuration taking into account all of the possible loss sources such as the integration loss caused by the NA mismatch between the ultra-low NA active fiber and the output fiber of the pump combiner. For that reason, the experimental setup has been installed based on a MOPA system as sketched in Fig. 6, which includes two stages; an oscillator in which the seed signal is produced, and an amplifier stage in which the signal coming from the seed is amplified to the high power levels. Firstly, the oscillator has been installed based on an Yb-doped active fiber having core and cladding diameters of $20 \mu \mathrm{m}$ and $400 \mu \mathrm{m}$, respectively.

The oscillator stage has been completed with an FBG pair consisting of a high reflective FBG having a reflectivity of $99.5 \%$ and output coupler FBG having a reflectivity of $10 \%$. Two pump diodes have been utilized in the oscillator stage as a pump source, and it has been integrated to a $6 \times 1: 1$ pump combiner having $20 \mu \mathrm{m} / 400 \mu \mathrm{m}$ fiber output. The total signal power produced at the end of the oscillator after the cladding light stripper (CLS) is around $\sim 150 \mathrm{~W}$. However, having passed through the $40 \mathrm{~m}$ long low NA active fiber, $110 \mathrm{~W}$ of this power is launched to the amplifier stage to be amplified. In the amplifier stage, the signal coming from the oscillator is amplified to the higher power levels by our $40 \mathrm{~m}$ long piece of fiber which has been pumped by six pieces of high power laser diode modules which are combined with a $6 \times 1: 1$ pump combiner having an output fiber having core/cladding diameters of $20 \mu \mathrm{m} / 400 \mu \mathrm{m}$ respectively. In addition, this fiber has a numerical aperture value of 0.06 . This information is critical since the fiber to be integrated has a numerical aperture of around 0.034 and a core diameter of $26 \mu \mathrm{m}$. Therefore, it can be said that the integration of the combiner fiber and our fiber is the most crucial part of this work since due to the NA mismatch between these two fibers some portion of the signal would be coupled to the cladding and hereby is lost in the CLS. Theoretically, the ratio of the power coupled to the cladding would be estimated based on the mode overlap between the two fibers and modal power distribution in these two fibers. In our case, the fibers have 0.034/0.06 NA values and $26 \mu \mathrm{m} / 20 \mu \mathrm{m}$ core diameters respectively. To accomplish this, we have built a far-field measurement setup using angular scanning technique to estimate the mode field diameters (MFD) of the fibers.

Based on the definition of MFD under Gaussian approximation, we have measured the angles at which the maximum 


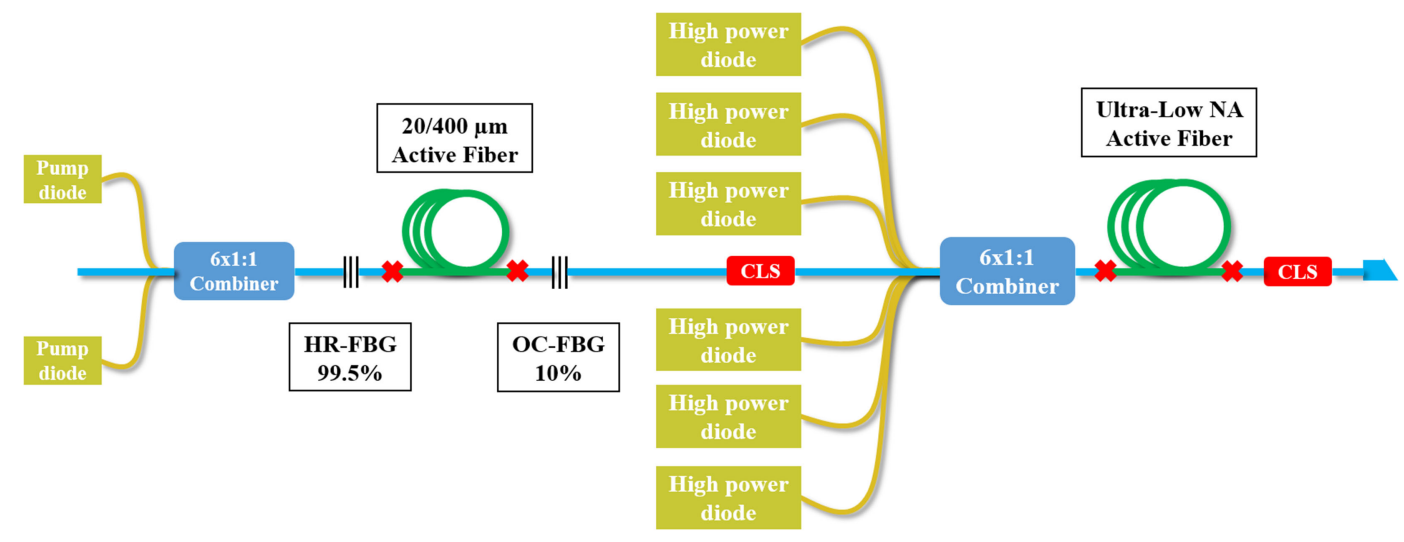

Fig. 6. Schematic representation of the fiber MOPA system that have built to examine the high power performance of the ultra-low NA fiber, HR-FBG, high reflective fiber Bragg grating; OC-FBG, output coupler fiber Bragg grating; CLS, cladding light stripper.

intensity drops to the $\mathrm{e}^{-2}$ for both fibers and obtained $3.5^{\circ}$ and $4.8^{\circ}$ respectively. Thus the coupling ratio can be estimated as $72.9 \%$ by the small angle approximation; $\tan (\theta) \sim \theta$. In real case, we have $110 \mathrm{~W}$ signal power reaching to the power meter after the ultra-low NA active fiber. With the help of a CLS integrated to the end of this fiber we have measured the power as $80 \mathrm{~W}$. Thus, the measured ratio would be $72.7 \%$ which is fairly close to the estimated one. Thereby, the signal power is going to be amplified in the amplifier stage is said to be $80 \mathrm{~W}$.

The average power of each high power diode module in the amplifier stage is around $250 \mathrm{~W}$ and so we had the total pump power of $1.5 \mathrm{~kW}$. However, the unabsorbed pump power is around $180 \mathrm{~W}$ at this pump power level. This corresponds to the $88 \%$ pump absorption approximately by $40 \mathrm{~m}$ long active fiber and this suggests that fiber length would be enough to absorb adequate pump power. Hereby, absorbed pump power can be calculated as $1320 \mathrm{~W}$. Since the pure laser power obtained after the CLS is $1050 \mathrm{~W}$, the slope efficiency of this laser system based on the absorbed pump power might be calculated as $75 \%$. According to the power characterization of the system $1.05 \mathrm{~kW}$ laser power is extracted in return for $1.32 \mathrm{~kW}$ absorbed pump power with a slope efficiency of $75 \%$ as sketched in Fig. 7(a). With the help of CLS, the unabsorbed pump power level is dramatically decreased, and as shown in Fig. 7(b), nearly $30 \mathrm{~dB}$ unabsorbed pump suppression is obtained. Fig. 7(b) also shows that the signal laser centered at $1079.66 \mathrm{~nm}$ was presented with a full width half at maximum (FWHM) of $1.64 \mathrm{~nm}$. As can be seen, there is also no sign for Raman signal at this power level. Additionally, we have also studied the output performance of our laser system in terms of temporal evolution, and no TMI occurs at this output power level as well. In this system, we have altered the bending diameter of the fiber from $1 \mathrm{~m}$ to $2 \mathrm{~m}$ with $50 \mathrm{~cm}$ steps but no significant change observed in the performance of the laser system.

Therefore, we have decided to bend the fiber in $1 \mathrm{~m}$ diameter and all of the data presented above belongs to this bending diameter. After completing high power performance test of the fiber, finally, we have measured the quality of the laser at the maximum power level, which is $1.05 \mathrm{~kW}$, by Thorlabs BP109-IR beam profiler and the data related is shown in Fig. 8. In addition
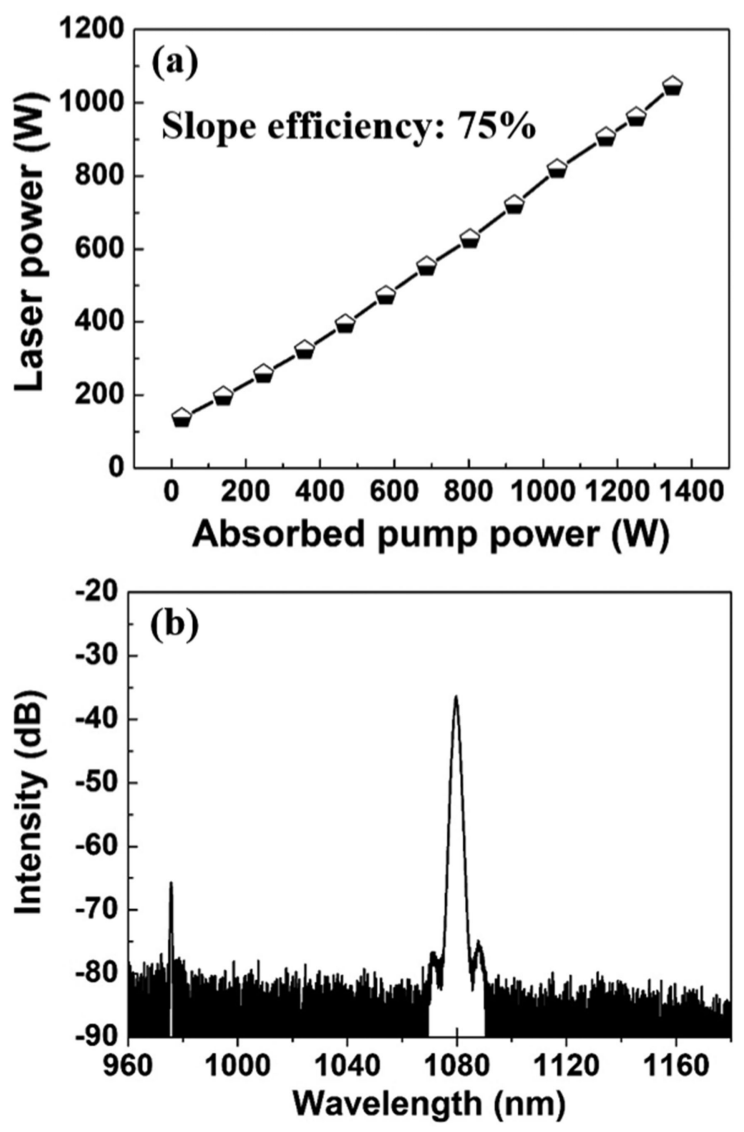

Fig. 7. (a) The power characterization of the ultra-low NA MOPA system up to $1.05 \mathrm{~kW}$ power level and (b) the optical spectrum at this power level.

to this, while the fiber is bent in $1 \mathrm{~m}$ diameter the beam profile image has been taken by using WincamD-UCD12-CCD beam profiler camera as shown in the inset of Fig. 8. The beam profile of the laser and the $\mathrm{M}^{2}$ result, which is 1.11 in $\mathrm{x}$-coordinate and 1.16 in y-coordinate, proves that the laser output has a good beam quality even at this large bending diameter. With this respect, this fiber is promising and it is improved multi-kW excellent beam quality fiber laser systems might be achieved based on these type of fibers in near future. 


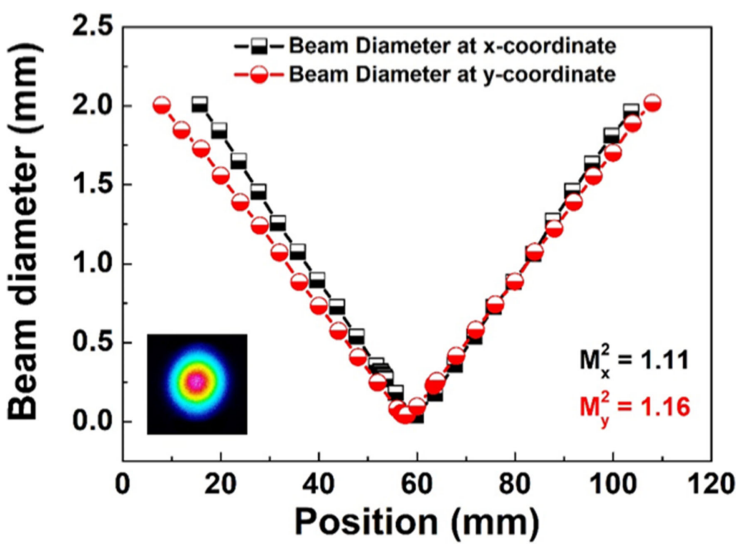

Fig. 8. Beam quality measurement of the ultra-low NA fiber MOPA system above $1 \mathrm{~kW}$ power level. Beam profile image of the laser output (inset).

\section{CONCLUSION}

In conclusion, we have fabricated an $\mathrm{Yb}$-doped LMA active fiber having an ultra-low NA of 0.034 and investigated both its preform and fiber properties. Having installed an NA characterization setup, we have verified the number we have predicted based on the RIP of the preform. After then, we have built a MOPA system to test the high power performance of this fiber up to power level above $1 \mathrm{~kW}$. Additionally, the beam quality measurement has been demonstrated at a power level more than $1 \mathrm{~kW}$, with $\mathrm{M}^{2}$ values 1.11 and 1.16 along with both $\mathrm{x}$ and $\mathrm{y}$ coordinates respectively. This information proves that the fiber has a good beam quality. Therefore, this preliminary work makes us believe that it may lead to multi-kW integrated monolithic all-fiber high-quality ultra-low NA fiber laser systems to meet the needs of the industry in the future.

\section{ACKNOWLEDGMENT}

The authors thank Elif Yapar Yıldırım, Ekin Teslime Balk, Bartu Simşek and Dr. Ali Karatutlu for their contributions to this study, and also to Seyit Ali Yaşar and Levent Ersoy for their technical support.

\section{REFERENCES}

[1] Y. Jeong, J. K. Sahu, D. N. Payne, and J. Nilsson, "Ytterbium-doped large-core fiber laser with $1.36 \mathrm{~kW}$ continuous-wave output power," Opt. Express, vol. 12, no. 25, Dec. 2004, pp. 6088-6092.

[2] K. Tankala et al., "New developments in high power eye-safe LMA fibers," Proc. SPIE, vol. 6102, pp. 610206-1-610206-9, 2006.

[3] D. Walton et al., "Kilowatt-level, narrow line width capable fibers and lasers," Proc. SPIE, vol. 6453, pp. 645314-1-645314-10, 2007.

[4] C. Jauregui, J. Limpert, and A. Tünnermann, "On the Raman threshold of passive large mode area fibers," Proc. SPIE, vol. 7914, pp. 791408-1-791408-6, 2011.

[5] D. P. Jablonowski, "Fiber manufacturing at AT\&T with the MCVD process," J. Lightw. Technol., vol. 4, no. 8, pp. 1016-1019, Aug. 1985.

[6] W. M. Flegal, E. A. Haney, R. S. Elliot, J. T. Kamino, and D. N. Ernst, "Making single-mode preforms by the MCVD process," AT\&T Tech. J., vol. 65, no. 1, pp. 56-61, Jan. 1986.
[7] C. Hupel et al., "MCVD based fabrication of low-NA Fibers for high power fiber laser application," in Proc. Adva. Solid State Lasers, Berlin, Germany, 4-9 Oct. 2015, pp. AM4A.2-1-AM4A.2-3.

[8] A. V. Smith and J. J. Smith, "Modeled fiber amplifier performance near the mode instability threshold," 2013. [Online]. Available: http://arxiv.org/ abs $/ 1301.4278$

[9] B. Ward, C. Robin, and I. Dajani, "Origin of thermal modal instabilities in large mode area fiber amplifiers," Opt. Express, vol. 20, no. 10, pp. 11407-11422, 2012.

[10] C. Jauregui, T. Eidam, H.-J. Otto, F. Stutzki, F. Jansen, J. Limpert, and A. Tunnermann, "Physical origin of mode instabilities in high-power fiber laser systems," Opt. Express, vol. 20, no. 12, pp. 12912-12925, 2012.

[11] R. Tao, X. Wang, and P. Zhou, "Comprehensive theoretical study of mode instability in high-power fiber lasers by employing a universal model and its implications," IEEE J. Sel. Top. Quantum, vol. 24, no. 3, 2018, Art. no. 0903319.

[12] Y. Jeong, A. J. Boyland, J. K. Sahu, S. Chung, J. Nilsson, and D. N. Payne, "Multi-kilowatt single-mode Ytterbium-doped large-core fiber laser," J. Opt. Soc. Korea, vol. 13, no. 4, pp. 416-422, 2009.

[13] D. Jain, Y. Jung, P. Barua, S. Alam, and J. K. Sahu, "Demonstration of ultra-low NA rare-earth doped step index fiber for applications in high power fiber lasers," Opt. Express, vol. 23, no. 6, pp. 7407-7415, 2015.

[14] F. Kong et al., "Large-mode-area fibers operating near single mode regime," Opt. Express, vol. 24, no. 10, pp. 10295-10301, 2016.

[15] V. Khitrov, J. D. Minelly, R. Tumminelli, V. Petit, E. S. Pooler, " $3 \mathrm{~kW}$ single-mode direct diode-pumped fiber laser," Proc. SPIE, vol. 8961, pp. 89610V-1-89601V-6, 2014.

[16] F. Beier et al., "Narrow linewidth, single mode $3 \mathrm{~kW}$ average power from a directly diode pumped Ytterbium-doped low NA fiber amplifier," Opt. Express, vol. 24, no. 6, pp. 6011-6020, 2016.

[17] F. Beier et al., "Single mode $4.3 \mathrm{~kW}$ output power from a diode-pumped Yb-doped fiber amplifier," Opt. Express, vol. 25, no. 13, pp. 14892-14899, 2017.

[18] K. Peng et al., "Single-mode large-mode-area laser fiber with ultralow numerical aperture and high beam quality," Appl. Opt., vol. 55, no. 35, pp. 10133-10137, 2016.

[19] S. Liu et al., "3 kW 20/400 Yb-doped aluminophosphosilicate fiber with high stability," IEEE Photon. J., vol. 10, no. 4, Aug. 2018, Art. no. 1503408.

[20] T. Deschamps, N. Ollier, H. Vezin, and C. Gonnet, "Clusters dissolution of $\mathrm{Yb}^{3+}$ in codoped $\mathrm{SiO}_{2}-\mathrm{Al}_{2} \mathrm{O}_{3}-\mathrm{P}_{2} \mathrm{O}_{5}$ glass fiber and its relevance to photodarkening," J. Chem. Phys., vol. 136, no. 1, pp. 014503-1-014503-4, 2012.

[21] S. Jetschke, S. Unger, A. Schwuchow, M. Leich, and J. Kirchhof, "Efficient $\mathrm{Yb}$ laser fibers with low photodarkening by optimization of the core composition," Opt. Express, vol. 16, no. 20, pp. 15540-15545, 2008.

Yakup Midilli received the B.Sc. degree in physics from Middle East Technical University, Ankara, Turkey, in 2012. He is currently working toward the Ph.D. degree with the National Nanotechnology Research Center (UNAM), Institute of Material Science and Nanotechnology, Bilkent University, Ankara.

Bülend Ortaç received the B.Sc. degree in physics from Karadeniz Technical University, Trabzon, Turkey, in 1997, the M.S. degree in teaching and diffusion of sciences and technology from ENS Cachan University, Paris, France, in 2000 , and the Ph.D. degree in optoelectronics from Rouen University, Rouen, France, in 2004. In March 2005, he joined the Institute of Applied Physics, FriedrichSchiller University, Jena, Germany, as a Postdoctoral Associate. Since November 2009, he has been a Research Assistant Professor with the Institute of Materials Science and Nanotechnology, Bilkent University, Ankara, Turkey. He is the Founder and the Principal Investigator of the Laser Research Laboratory. He has authored or coauthored more than 100 research articles in major peer-reviewed scientific journals (more than 50) and conferences (more than 90) in the field of laser physics. His research interests include the development of powerful fiber lasers in the continuous-wave regime to pulsed regime (ns, ps, and fs) and the demonstration of laser systems for real world applications. And also he has recently been selected as an OSA Senior Member. 